\title{
Théologiques
}

\section{Dieu est-il interdit?}

\section{Denise Couture}

Volume 6, numéro 2, octobre 1998

Dieu interdit

URI : https://id.erudit.org/iderudit/024966ar

DOI : https://doi.org/10.7202/024966ar

Aller au sommaire du numéro

Éditeur(s)

Faculté de théologie de l'Université de Montréal

ISSN

1188-7109 (imprimé)

1492-1413 (numérique)

Découvrir la revue

Citer cet article

Couture, D. (1998). Dieu est-il interdit? Théologiques, 6(2), 111-118.

https://doi.org/10.7202/024966ar d'utilisation que vous pouvez consulter en ligne.

https://apropos.erudit.org/fr/usagers/politique-dutilisation/ 
Théologiques 6/2 (1998) 111-118.

\section{Dieu est-il interdit?}

Denise COUTURE

Faculté de théologie

Université de Montréal

Les cinq années d'existence de la Revue ont été l'occasion d'évaluer la réalisation de son premier objectif, l'interdisciplinarité entre la théologie et les autres sciences humaines. On sait que l'interdisciplinarité signifie plus que la juxtaposition de travaux, sur un même thème, rédigés par des spécialistes de différentes disciplines. À la Revue, elle s'est réalisée également dans des corps-à-corps, soit entre les membres du Comité de rédaction lors de la problématisation des différents numéros, soit entre les signataires des articles publiés dans le cadre des débats-midi de Théologiques, tenus après la parution des numéros. Au bilan, on a commencé à pratiquer une interdisciplinarité entre sciences humaines et théologie; on l'a encore peu pensée. Le présent numéro reprend la question, de façon originale, je crois, par le choix de ses actants et actantes et par l'option d'une démarche d'exploration.

On m'a confié la tâche de faire une synthèse des sept contribu tions de ce numéro, qui se présente comme le résultat d'un projet spécial du Comité de rédaction et dont les articles ont été rédigés par les membres de l'équipe. Sa problématique est celle de l'effacement de Dieu au sein du discours universitaire, repéré certes en sciences humaines, mais également en théologie même. Il s'agissait d'habiter, ensemble, "une question préalable non encore acquise " (Liminaire, p. 3) qui ne relève ni d'une problématique intra-théologique, ni d'un questionnement habituel en sciences humaines. Non spécialistes de la question, les membres du Comité de rédaction ont accepté de prendre un risque et de présenter, dans ce numéro, une première analyse du "Dieu interdit " à l'intérieur de leur propre discipline ou de leur champ de spécialisation. Le résultat de cette exploration soulève diverses questions sur les rapports entre les théo- 
logies et les sciences humaines. Je ferai ressortir les principales thèses des auteurs et des auteures.

Gilbert Renaud analyse l'effacement de Dieu en sciences humaines appliquées, plus particulièrement en service social. En contexte québécois, c'est dans les années 1960 que Dieu disparaît définitivement dans ce secteur de connaissance. L'émancipation du discours religieux et clérical fut ressentie comme la libération d'un joug; elle ouvrait à la légitimation scientifique du discours. Le développement des processus de recherche s'est par la suite orienté dans la direction des sciences humaines appliquées. Guidé par le principe d'efficacité, il a été marqué par une pensée positive, une technocratisation des sociétés et une instrumentalisation de l'existence humaine.

Acquises sur le fond d'une émancipation du religieux, ces lignes de développement de la recherche scientifique auraient un double effet, celui, sur les sciences humaines, d'occulter la question de " toute finalité transcendantale » qui semble pourtant ne pas pouvoir ne pas resurgir au sein des processus de rationalisation et, celui, inverse, sur la théologie, de la pousser, à son tour, vers la gestion sociale rationalisée. L'interdit Dieu ne frapperait-il pas ainsi les sciences humaines appliquées comme la théologie?

G. Renaud débutait son article en distinguant deux approches de l'interdisciplinarité entre la théologie et les sciences humaines. D'abord, pour un chercheur en sciences humaines, la question de Dieu semble relever du personnel et du privé, non du scientifique. Une véritable interdisciplinarité avec la théologie paraît alors impossible, sinon dans un rapport à sens unique, et dans le sens d'une contribution des sciences humaines à la théologie. Est-ce ce qui se passe? Une autre perspective situerait la question de Dieu au fondement de la théologie et pourrait ouvrir à une véritable fécondation réciproque des discours « qui n'occulte pas, mais reconnaît et accepte plutôt le fondement différencié des sciences humaines et de la théologie" (p. 8). Les sciences humaines appliquées se trouvent devant la tâche de répondre à la résurgence de la question du sens de l'exis tence humaine. La théologie peut-elle contribuer en posant la question de Dieu dans la direction de la quête de sens ultime?

L'analyse de Nicole Laurin, en sociologie, rejoint celle de G. Renaud sur certains aspects, mais il est intéressant de noter 
qu'elle ouvre à une autre position sur l'interdisciplinarité entre les sciences humaines et la théologie. Je retiens d'abord ses remarques sur la particularité du contexte québécois en regard de notre question. Au Québec, le catholicisme social y a influencé une sociologie, enseignée alors par des clercs, jusque dans les années 1960. Par la suite, "un fossé infranchissable se creuse entre l'Église et l'institution universitaire, exception faite des facultés de théologie » (p. 31). Ainsi, on ne trouvera pas, comme en France, une figure de "l'intellectuel catholique ». Pratique scientifique et foi religieuse se repoussent, au contraire, jusqu'à être "jugées radicalement incompatibles" (p. 31).

Mais l'interdit Dieu, en sociologie, n'est pas seulement ni d'abord lié au retrait de l'influence de courants religieux de pensée sur les sociologues. Il est inscrit dans un mode de savoir. Selon N. Laurin, la sociologie n'a rien à dire de Dieu en tant "qu'hypothèse fondatrice "; elle n'en a nul besoin. L'auteure montre l'effacement de Dieu tant dans la théorie générale de la sociologie que dans le champ spécialisé des sociologies de la religion. Ce n'est pas que le vocable ne circule plus dans les discours de la sociologie mais, mué en objet soumis au savoir spécifique de la discipline, on le traite comme une « réalité sociale » parmi les autres.

À une théologie qui vise à construire un dialogue interdiscipli naire avec la sociologie, celle-ci ne peut offrir que sa manière de regarder, sans Dieu, la société. La «pesanteur» du fait social, ajoute N. Laurin, « n'appartient pas au déterminisme " et " ne s'oppose pas à la liberté. Pour la sociologie, il n'est en effet ni véritable déterminisme, ni véritable liberté »(p.32). Cette analyse, me semble-t-il, met en question une pratique théologique, dite d'interdisciplinarité avec la sociologie, qui veut intégrer des résultats de la sociologie à ce qui demeure interprété, en théologie, comme une histoire humaine en vue d'une liberté en Dieu. Dans son compagnonnage avec les sciences humaines, devenu habituel, la théologie a-t-elle pris acte de «l'interdit Dieu " qui appartient à ses approches théoriques?

Walter Moser, du département de littérature comparée, propose l'hypothèse d'un "trou-en-forme-de-Dieu " qu'un système discursif doit combler, et qu'il comble, par une appropriation des "structures discursives originairement théologico-religieuses " (p. 35). Du Dieu du discours théocentrique, l'auteur retient qu'il est l'alpha et 
l'oméga, le Super-sujet, qu'il fonde toutes choses. On lui attribue les prédicats les plus totalisants et on lui fait occuper en même temps des positions opposées (transcendant et immanent, absent et présent, et autres). Aucun mot ne saurait le dire ou l'expliquer (non-prédicabilité). Dans la structure actantielle, "Dieu peut occuper toutes les positions de la structure $"$ (p. 38). Tout lui est possible.

Dans le processus de la sécularisation, de la disparition de l'instance Dieu, W. Moser repère des déplacements dans les mises en discours de cette instance. De nouvelles occuperaient son statut et sa fonction. L'auteur propose, à titre d'exemples, les notions de pouvoir et d'État en politique, de capital en économie et de désir en psychanalyse, du moins telles que réemployées couramment hors des contextes rigoureux de leurs articulations disciplinaires. Elles ont un statut d'instance ultime et suprême dans l'ordonnance discursive et on peut leur attribuer les caractéristiques ci-haut mentionnées du Dieu congédié. Sur le plan de la mise en discours, elles occupent la fonction laissée vacante par le "trou-en-forme-de-Dieu». Il y aurait résurgence de l'instance du Dieu pré-moderne par mode de déplacement, mais aussi multiplication, puisque chaque secteur particulier a un concept fondateur qui comble un espace laissé vacant. L'État serait la forme métarmorphosée la plus reconnaissable de l'instance divine. W. Moser suggère d'autres formes de déplacements de l'ins. tance Dieu vers ce qu'il appelle des "figures anthropomorphes", tels le Philosophe des Lumières, le créateur artistique, les scientifiques sur les premières lignes des découvertes technologiques, le pédagogue ou l'homme politique. La mise en discours de l'instance Dieu serait ainsi multiple et diversifiée, sous les yeux même de ceux et celles qui pensent l'avoir congédié pour de bon.

Cette analyse m'amène à demander si le déplacement discursif en vue de combler le «trou-en-forme-de Dieu » ne donne pas au Dieu de la théologie la position d'un dieu parmi les autres dieux des discours. Ce Dieu ne correspond pas au Dieu pré-moderne. Pourraiton aller jusqu'à dire, sur la base de l'analyse de W. Moser, qu'il ne serait plus ni le plus grand, ni le plus fondamental, mais l'une des instances modernes du Dieu mutant?

Les trois premiers articles confirment l'effacement de Dieu dans le discours universitaire des sciences humaines. Ils manifestent, à mon avis, trois attitudes ou postures qui nous traversent tour à tour 
devant cet effacement: une forme de nostalgie; un rappel de sa nécessité et de ses implications; ou le soupçon de déplacements discursifs qui camoufflent l'omniprésence de l'instance dite évanouie. Le caractère problématique de la mise en discours de Dieu ressort différemment dans les quatre articles suivants, écrits par des professeurs œuvrant à la Faculté de théologie. Chacun pointe, d'une manière ou d'une autre, l'un des axiomes de la théologie moderne, à savoir le danger, en théologie, de faire parler Dieu.

Olivette Genest identifie une paradoxale juxtaposition d'omniprésence et d'absence de Dieu dans le discours de l'exégèse biblique. Omniprésence, car le travail porte sur un texte saturé de Dieu, mais, absence, car de Dieu, l'exégèse ne parle peut-être pas. L'approche scientifique de l'exégèse biblique appartient aux sciences humaines et peut relever même d'une science exacte. Elle se meut souvent dans ce que l'auteure appelle "l'anti-chambre de l'interprétation ", ce qui ne va pas sans poser la question de la neutralité du lecteur ou de la lectrice. Sur la base d'une analyse de la production discursive dans le champ de l'éxégèse biblique, $O$. Genest note que les exégètes parlent peu de Dieu. D'ailleurs, telle ne serait pas leur tâche, qui consisterait plutôt à préparer un chemin vers un rapport au texte qui laisse le texte parler de Dieu. Elle doit laisser à l'auditrice ou à l'auditeur la tâche d'ouvrir la relation personnelle à Dieu. Cette relation ne relève plus de l'enseignement exégétique, mais de la lectio divina. De quel Dieu parlerait l'exégèse parmi les figures de Dieu dans le texte biblique? $\grave{A}$ partir de quelle perspective contemporaine qui serait à élucider? En vue de quel public qui a, déjà, ses nombreux dieux? Dieu « demeure une interrogation qui intrigue, un souci qui passe et revient, une attente, une mouvance » (p. 58). Le rapport à Dieu, à sa présence, ne saurait être traité dans le cadre de la rationalité scientifique à laquelle appartient l'exégèse biblique.

Le texte d'O. Genest soulève la question des rapports entre le personnel et le scientifique, un rapport, ici, d'exclusion réciproque. G. Renaud notait également dans son texte cette possible interprétation. La chose est encore à penser. On a suggéré avec profit que si le personnel est politique, il est aussi théorique.

Guy Lapointe, en liturgie chrétienne, répond à la question de l'effacement de Dieu par une question : « Comment peut-on simplement imaginer que Dieu pourrait ne pas être l'acteur incontournable 
de toutes nos liturgies?" (p. 61) En liturgie, le problème Dieu ne serait pas celui de sa disparition, mais, à l'inverse, celui d'une possession dans un surcroît de présence. Cependant, la liturgie est devenue une pratique où Dieu n'est pas seulement rendu présent, mais possédé et approprié. Le "risque de la liturgie", selon G. Lapointe, est qu'elle devienne objet de consommation et, ainsi, donne lieu à une mainmise sur Dieu, plutôt que d'ouvrir à l'espace symbolique, à une dépossession, à une dé-prise. Le théologien liturgiste montre que la dimension symbolique de la liturgie appartient à l'espace non conceptuel et à la non maîtrise; la liturgie serait un lieu-transit de non appropriation qui se joue dans une absence-présence d'un Dieu qui demeure interrogation.

L'auteur fait un rapprochement entre cette problématique, en liturgie, et la position de la théologie dans le monde universitaire. Le peu de crédibilité qu'on accorde parfois à celle-ci ne relève-t-il pas d'une perception voulant qu'on y pratique une "apologétique de la Vérité confessée » (p. 62)? La théologie serait perçue comme rendant Dieu plus-que-présent et travaillant, d'une position confessionnelle, sur l'affirmation de Dieu. Pour Guy Lapointe, la théologie aborde plutôt " la question de Dieu (...) comme un discours sur le problème de Dieu, même si ce discours se situe dans une tradition croyante " (pp. 62-63). Elle pose la question de Dieu et la situe dans l'histoire de sa mise en scène.

Jean-Guy Nadeau demande si la "théologie pratique " ne participe pas au tabou excluant Dieu dans les sciences humaines et sociales. Il analyse les travaux produits dans ce secteur de la théologie et distingue deux approches. L'une aborde Dieu le plus souvent à la manière des autres sciences humaines, comme objet ou concept langagier. En effet, les théologies pratiques empiriques et fondamentales font peu de place à Dieu. Dieu y apparaît comme « objet de croyance ", traité à la manière de la sociologie ou de la psychologie de la religion, ou comme un concept, une occurrence langagière et discursive, traité comme en linguistique. L'autre approche assigne à Dieu une fonction de référence ultime. Les théologies pratiques critiques, des théories politiques engagées, ainsi que les théologies pratiques pastorales ou programmatiques donnent à Dieu la position de fondement, soit en terme de référence ultime, prophétique et axiologique, soit en terme de Sujet de l'histoire qui convoque et appelle. 
Ces termes ne sont pas sans rappeler les caractéristiques du Dieu congédié, tel qu'analysé par W. Moser.

Pour J.-G. Nadeau, la théologie ne relève ni du paradigme empirique des sciences humaines, ni du paradigme métaphysique et déductif de la théologie classique, mais se situe entre les deux. L'auteur semble ici répondre à l'appel de son collègue G. Renaud : la théologie pose " les questions du sens ultime et du cheminement vers l'Être que le rationalisme a laissées à l'herméneutique, à la métaphysique et à la théologie " (p. 93).

Enfin, Jean-Claude Petit pose la question du Dieu interdit à partir du champ d'étude de la théologie fondamentale. De l'histoire des énoncés sur Dieu, dans la tradition chrétienne, il retient un courant de pensée représentée par Nicolas de Cues. Il aurait la force "de redonner un sens chrétien à cette question de Dieu " (p. 102). Selon cette tradition, on ne rencontre Dieu, qui demeure "totalement inaccessible ", que sur le chemin de soi-même vers soi-même, et non dans un oubli de soi. Le discours sur Dieu n'a de sens qui "s'il se déploie comme une véritable "question" "(p. 102), comme ce qui met en chemin dans l'ouvert d'une question qui demeure ouverte, au sein d'un écart entre moi et moi. Sur les traces de Paul Tillich et de la théologie du XX $\mathrm{XX}^{\mathrm{e}}$ siècle, J.-C. Petit montre que l'articulation fondamentale de la question de Dieu est indissociable de la question anthropologique. Il y a un "absolute concern", il y a la question de Dieu, à partir de la distance, de la faille, entre soi-même et soimême.

"On parle beaucoup de Dieu en théologie" (p. 105). Avec assurance. On oublie qu'on ne peut pas parler à Dieu pour en tirer des réponses. La théologie n'a pas à combler l'entaille d'inquiétude propre à l'humain. La théologie devrait parler sans tapage et à voix basse. Elle « n'a pas à parler plus fort que Dieu " (p. 109).

Les analyses confirment l'effacement de Dieu dans le discours des sciences humaines, du moins du Dieu comme fondement ultime et transcendant. On lui a donné congé en sciences humaines appliquées, en sociologie, en sciences humaines tout court et dans certaines approches en exégèse biblique et en théologie pratique. Si le vocable Dieu circule encore, c'est surtout comme objet d'analyse empirique, par le biais de la question des croyances. 
Un des problèmes relevés par les théologiens est à l'inverse celui d'un surplus de présence de Dieu en théologie. Les théologiens semblent demander à la théologie de rendre Dieu à un silence, à lui approprié.

Je retiens l'invitation de G. Renaud à construire un discours qui ne tue pas la question de la finalité transcendantale et à laquelle répondent assez naturellement les trois théologiens, G. Lapointe, J.G. Nadeau et J.-C. Petit; les appels de N. Laurin et d'O. Genest à penser jusqu'au bout les implications d'un discours «sans Dieu » en sciences humaines; et la question des déplacements discursifs de W. Moser qui pourrait conduire à penser le Dieu des théologiens, non plus comme l'alpha et l'oméga prémodernes, mais comme un dieu parmi les nouveaux dieux mutants des discours modernes. 